\title{
Update on state of the art magnetic resonance angiography techniques
}

This article was published in the following Dove Press journal:

Journal of Vascular Diagnostics

15 May 2015

Number of times this article has been viewed

\author{
Waleed Brinjikji \\ Phillip M Young \\ Department of Radiology, Mayo Clinic, \\ Rochester, MN, USA
}

\begin{abstract}
Magnetic resonance angiography (MRA) is a dynamic and growing field. In most cases, MRA has replaced conventional angiography and computed tomographic angiography in the evaluation of vascular disease. Numerous MRA techniques are available in clinical practice, each with their own strengths and weaknesses. A number of non-contrast techniques are available that do not expose patients to the potentially toxic effects of gadolinium. Dynamic time-resolved imaging is emerging as an important tool in the evaluation of the hemodynamic consequences of vascular disease. In this review, we summarize the state of the art in MRA of the aorta and peripheral arteries.
\end{abstract}

Keywords: magnetic resonance angiography, aorta, renal arteries, mesenteric arteries, peripheral arteries

\section{Introduction}

Magnetic resonance angiography (MRA) is a dynamic and growing field. In most cases, MRA has replaced conventional angiography and computed tomographic angiography (CTA) in the evaluation of vascular disease. Numerous MRA techniques are available in clinical practice, each with their own strengths and weaknesses. One exciting facet of MRA is the ability to obtain time-resolved imaging allowing for evaluation of the hemodynamic effects of various vascular lesions. MRA also allows for high-resolution angiographic images with and without the use of intravenous contrast, thus allowing for improved patient safety, especially among those with chronic renal disease. In this review, we discuss some commonly used and state-of-the-art MRA techniques and their exciting applications in the diagnosis and treatment of vascular disease.

\section{MRA techniques Contrast-enhanced MRA}

Contrast-enhanced (CE)-MRA is the gold standard MRA technique as it allows for acquisition of large-volume data sets in coronal and/or sagittal planes in a single breath hold. ${ }^{1,2}$ CE-MRA with gadolinium-based contrast agents is preferred. To guarantee accurate bolus timing, our practice generally employs automated detection of the contrast material and real-time depiction of bolus arrival for neurologic applications, although we sometimes use the test bolus technique for body applications. ${ }^{3,4}$ Magnetic resonance-compatible power injectors allow precise injection of the gadoliniumbased material using predefined rates and volumes, improving intravascular contrast enhancement. ${ }^{3,4}$ Generally, a bolus of at least $0.1 \mathrm{mmol}$ gadolinium $/ \mathrm{kg}$ body weight is required in three-dimensional (3D) CE-MRA.
Correspondence: Phillip M Young Department of Radiology, Mayo Clinic, 200 Ist Street SW, Rochester, MN 55905, USA

Email young.phillip@mayo.edu (c) (i) (5) 2015 Brinijkji and Young. This work is published by Dove Medical Press Limited, and licensed under Creative Commons Attribution - Non Commercial (unported, v3.0) permission from Dove Medical Press Limited, provided the work is properly attributed. Permissions beyond the scope of the License are administered by Dove Medical Press Limited. Information on
how to request permission may be found at: http://www.dovepress.com/permissions.php how to request permission may be found at: http://www.dovepress.com/permissions.php 
CE-MRA images are performed with 3D gradient echo sequences using short repetition times and echo times., ${ }^{2,5}$ A large field of view is typically used. The slab volume can be acquired in any plane of orientation. ${ }^{2,5}$ Each dataset can be acquired in a single breath-hold period using short acquisition times. Fat saturation pulses or water-fat separation techniques can be helpful to suppress high signal intensity of surrounding fat. ${ }^{2,5}$ Post processing methods include multiplanar reformats, maximum intensity projection, shaded surface display, and volume rendering. Multiplanar reformats are used as problem-solving tools because they are easily performed with commercially available software. A second approach to 3D MRA is to perform time-resolved imaging with temporal interpolation. Temporal interpolation is a process in which the low spatial frequency data within the center of k-space is sampled more frequently than the higher spatial frequency data located more peripherally. The main advantage of this technique is high temporal resolution and image quality, even in the setting of motion and poor breath holds. ${ }^{6}$

\section{Non-contrast MRA}

The most popular techniques for unenhanced MRA include time of flight (TOF) and phased contrast imaging. ${ }^{7-9}$ TOF MRA allows for adequate evaluation of vascular pathology in a majority of cases. Disadvantages of TOF include a long acquisition time and signal loss of in-plane flow resulting in overestimation of the degree of stenosis in some cases. ${ }^{7-9}$ Long acquisition times for TOF imaging make these imaging sequences susceptible to motion artifact, thus limiting diagnostic accuracy in many cases. ${ }^{7-9}$ On the other hand, phase-contrast (PC)-MRA is independent of flow direction. Acquisition times are generally shorter than for TOF MRA. PC-MRA provides excellent contrast and spatial resolution. Limitations to PC-MRA include loss of signal in vessels with turbulent flow and the susceptibility to motion artifacts. ${ }^{7-9}$

Another effective non-contrast MRA technique is electrocardiogram (ECG)-gated fast spin echo MRA. Fast spin echo MRA produces bright blood MRA images by taking advantage of the flow void effect of fast systolic arterial flow. ${ }^{7-9}$ Advantages of fast spin echo MRA include less susceptibility to field heterogeneities, sensitivity to slow flow, and a short imaging time. Motion artifact is a major limitation of this technique. Steady-state free precession (SSFP) angiography is another effective non-contrast imaging technique. Image contrast is T2/T1-weighted, resulting in high signal intensity of blood..$^{7-9}$ The major advantage to this technique is short acquisition times and a high signal-to-noise ratio. This technique has been applied in the aorta, specifically with ECG gating in the thoracic aorta as well as in children with congenital heart disease where the technique can be used with a respiratory navigator to provide high-resolution images of the coronary arteries. ${ }^{10}$ SSFP MRA images are improved with breath hold. Limitations of SSFP MRA include the fact that there is usually little flow dependence of these sequences, as well as susceptibility to field heterogeneities. These sequences can be used to follow aneurysm size, for example, but may underestimate or even miss an intravascular thrombus.

Quiescent inflow single shot (QISS) MRA is another emerging non-contrast MRA technique. This technique uses ECG-triggered, fat-suppressed two-dimensional (2D) SSFP sequences with a quiescent interval to allow for enhancement of inflowing blood. ${ }^{7-9,11}$ QISS MRA allows for faster imaging times than conventional TOF MRA, as it requires less tailoring to patient factors such as heart rate, arrhythmia, or slow flow, and is less sensitive to motion. ${ }^{7-9,11}$

\section{Flow magnetic resonance imaging}

Velocity-encoded cine (VEC) magnetic resonance imaging (MRI) is a time-resolved imaging modality that can be performed using phase-contrast sequences. In general, phase-contrast sequences can be $2 \mathrm{D}$ or $3 \mathrm{D} .{ }^{12-14}$ Imaging is dependent on a velocity-encoding (VENC) parameter. The VENC determines the range of blood flow velocity that can be measured. For example, if the VENC is set at $200 \mathrm{~cm} / \mathrm{sec}$, velocities of up to $\pm 200 \mathrm{~cm} / \mathrm{sec}$ can be measured. Velocities greater than the VENC result in aliasing artifact, which is difficult to correct. Sensitivity decreases with higher VENC. ${ }^{12-14}$ Two-dimensional velocity VENC MRI can be used to measure blood flow at any position of the aorta and any of its branches. It is possible to acquire one position within a breath hold with a temporal resolution of about $30 \mathrm{msec}$ depending on the heart rate and other imaging parameters. A higher temporal resolution of about $10 \mathrm{msec}$ can be attained with free breathing or breath-gated acquisitions. Two-dimensional VEC MRI has been demonstrated to provide accurate blood flow measurements in pediatric and adult pathologies as well as within endovascular stent grafts. ${ }^{12-14}$

Recent developments have resulted in a relatively fast $3 \mathrm{D}$ phase-contrast MRI sequence (3D VEC MRI, known also as four-dimensional [4D] flow MRI), which allows for acquisition of the entire velocity field in about 20-30 minutes. ${ }^{12-14}$ With appropriate image processing, vector fields, streamlines, and other parameters illustrating the changing velocity field during the cardiac cycle can then be displayed in a cine fashion. ${ }^{12-14}$ 


\section{MRI contrast agents}

MRI contrast agents affect the relaxation times of nearby protons. Gadolinium is by far the most commonly used MRI contrast agent available. ${ }^{1,15-18}$ Free gadolinium is very toxic. ${ }^{19}$ Chelation of gadolinium by ligands significantly reduces its acute toxicity. A wide variety of gadolinium agents are in use today. Gadolinium agents are classified as ionic-linear ligand, non-ionic-linear ligand, ionic-cyclic ligand, and non-ionic-cyclic ligand. ${ }^{20}$ Linear chelates have flexible open chains that do not have strong bonding to gadolinium. The most stable gadolinium contrast agents are the macrocyclics and the least stable are the non-ionic-linear chelates such as gadodiamide and gadoversetamide.

Gadolinium contrast agents are given by bolus injection and have pharmacokinetics similar to those of iodine-based radiographic contrast agents. Gadolinium contrast agents are generally excreted by passive glomerular filtration without secretion or reabsorption. Elimination is typically complete in 24-48 hours. In patients with normal renal function, nearly $100 \%$ of these agents are excreted within 24 hours of injection. Gadofosveset is eliminated through both renal and hepatobiliary pathways. ${ }^{21,22}$

In general, gadolinium contrast agents are considered to be safer than iodinated contrast agents due to the rarity of life-threatening contrast reaction and the relatively decreased nephrotoxicity of gadolinium compared with iodinated contrast agents. However, gadolinium contrast agents are not without their risks. Patients with reduced renal function are at risk of nephrogenic systemic fibrosis. Nephrogenic systemic fibrosis is a sometimes fatal fibrosing disease which involves the skin and subcutaneous tissues but can also affect the lungs, esophagus, heart, and skeletal muscles. ${ }^{21,22}$ In general, gadolinium should not routinely be administered to patients with a glomerular filtration rate of $<30$, and stringent risk-benefit analysis should govern administration in these patients..$^{21,22}$ Another concern with gadolinium agents is uptake in the basal ganglia and dentate nucleus. One recently published study demonstrated that patients receiving multiple gadolinium boluses have higher T1 signal intensity on pre-gadolinium images in their dentate nucleus and basal ganglia, presumably secondary to gadolinium deposition. The clinical significance of these findings has not been established. ${ }^{23}$

\section{Imaging applications in pathology Thoracic aortic aneurysms}

In evaluation of thoracic aortic aneurysms, ECG-gated gradient-echo 3D MRA is the preferred method due to high isotropic spatial resolution. However, some studies suggest that non-contrast, 3D SSFP MRA with ECG gating can provide superior image quality with improved diagnostic accuracy in measurements of thoracic aortic aneurysms. ${ }^{24}$ One advantage of this technique over CE-MRA is that CE-MRA has to fit within one breath hold and has to be completed after the first pass of the contrast agent, whereas native MRA is collected over many heart beats and is independent of the passage of contrast media. Further, there is less motion artifact affecting frequently pulsatile structures, such as the aortic root, with 3D ECG-gated techniques.

Aneurysms of the thoracic aorta have been shown to be associated with complex abnormal helical flow patterns that are thought to play a role in the progression of aneurysms. ${ }^{25}$ Four-dimensional flow MRI has also proven useful in the presurgical staging of patients with aortic valve disease. ${ }^{26}$ Aortic valve disease is associated with post-stenotic dilatation of the ascending aorta. ${ }^{27,28}$ Studies assessing flow jets in patients with aortic valve stenosis have demonstrated that these patients have eccentric flow jets with stenotic and deformed aortic valves. Flow eccentricity can be quantified and has been shown to correlate with focally elevated wall shear stress and aortic dilatation, factors associated with aneurysm development. ${ }^{27,28}$ For example, Hope et al found that patients with bicuspid aortic valves and eccentric systolic ascending aortic blood flow had significantly and asymmetrically elevated wall shear stress, potentially placing these patients at higher risk of ascending aortic aneurysm. ${ }^{29}$ Four-dimensional flow MRI could thus potentially be a useful tool for risk stratification of patients with aortic valve disease with regard to aneurysm development.

Advanced MRA techniques are essential in evaluation of aneurysms of the descending aorta as well. MRA is often used to select patients who undergo thoracic endovascular aortic repair. Challenges faced by endovascular surgeons in assessing aortic aneurysms include proper evaluation of aortic arch angulation, determining the size of proximal and distal landing zones, evaluation of true and false lumen in type B aortic dissections, and evaluation of branch vessels. ECG-gated MRA has a number of advantages in that it allows the surgeon to appreciate the changes in size of the anatomical areas of interest during the cardiac cycle with high spatial resolution. However, this high spatial resolution comes at the expense of long imaging and post-processing times..$^{25}$ Four-dimensional PC-MRA is another emerging technique in the evaluation of descending thoracic aortic aneurysms.

\section{Acute aortic syndromes}

MRA has very high accuracy in evaluation of aortic dissection. CE-MRA is the gold standard MRA technique 
used in evaluation of aortic dissection due to its high spatial and contrast resolution and its accuracy in detection of branch vessel involvement. ${ }^{30,31} \mathrm{CE}-\mathrm{MRA}$ images can be rapidly acquired without the need for ECG triggering. CE-MRA allows for accurate assessment of the anatomy of aortic dissection with high signal-to-noise ratios. However, bolus timing is necessary for accurate assessment. With CE-MRA, the intimal flap is seen as a dark line within the two lumens.

A number of non-contrast MRA techniques are helpful in evaluation of aortic dissection. Black blood sequences covering the aorta allow for adequate contrast between the aortic lumen and the vessel wall layers. With these imaging sequences, the dissection flap appears as a dark linear thin intraluminal structure. The true lumen can be differentiated from the false lumen in that the true lumen is usually smaller and shows signal void, whereas the false lumen has a higher signal intensity due to clot and turbulent/slow flow (Figure 1). ${ }^{30}$

Functional imaging with dynamic sequences allows for improved evaluation of how the dissection affects aortic hemodynamics and possible aortic regurgitation, and evaluation of clot burden. Phase-contrast imaging can be used for evaluation of inflow and outflow patterns through both the true and false lumen. The patent false lumen of chronic dissections has been shown to have slower helical or laminar flow than the true lumen. Helical flow has been shown to be associated with progression of disease. ${ }^{32,33}$ In patients with aortic dissection, 4D PC-MRA allows for accurate visualization and quantification of flow characteristics and gives valuable information about stroke volume, velocity, dominant proximal and distal tears, and helical flow. ${ }^{25}$ PC-MRI is also helpful in understanding how dissections affect the hemodynamics of aortic branch vessels. ${ }^{30}$ Evaluation of aortic hemodynamics has been shown to be helpful in preoperative planning for patients undergoing thoracic endovascular aortic repair.

In patients with intramural hematoma, MRI can be used to assess the acuity of the hematoma due to changes in the

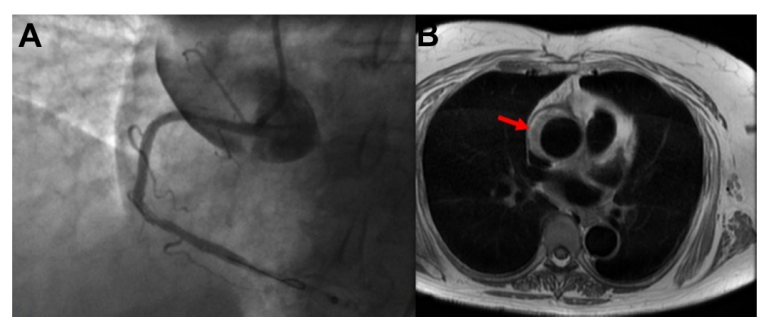

Figure I Intramural hemorrhage.

Notes: (A) Right coronary angiogram demonstrating a dissection extending to the aortic root. (B) Black blood sequence demonstrates a large subintimal dissection with hyperintense hemorrhage consistent with aortic dissection (red arrow). signal characteristics of hemoglobin degradation products. ${ }^{34}$ In general, intramural hematoma is demonstrated by wall thickening and mural hyperintensity on T1-weighted black blood images. In the hyperacute phase, intramural hematoma may be isointense on T1-weighted images and hyperintense on T2-weighted images. Black blood images are also used in evaluation of penetrating atheromatous ulcer. Imaging reveals intimal disruption with extension of the ulcer into the thickened tunica media. ${ }^{30}$

\section{Cryptogenic stroke}

MRA is a valuable tool in evaluating the presence of atheromatous plaque that could be the underlying cause of cryptogenic stroke. ${ }^{35}$ Descending thoracic aortic plaques are conventionally thought to be an embolic source of stroke in patients who have severe aortic valve insufficiency causing retrograde flow and embolization in case of plaque rupture. Because the descending aorta is distal to the left subclavian artery and retrograde embolization is thought to be unlikely, these plaques have not usually been considered a potential source of stroke. While transesophageal echocardiography is considered the historical gold standard for evaluation of aortic arch plaque, MRI and MRA have emerged as superior imaging modalities. Aortic atheromatous disease is present in up to $20 \%$ of patients with stroke or transient ischemic attack. ${ }^{36}$ Non-contrast and contrast-enhanced techniques can be used to assess for plaque burden as well as the presence of ulcerated plaque. ${ }^{37}$

Four-dimensional PC-MRI has been shown to be an effective tool for the evaluation of cryptogenic stroke. ${ }^{37,38}$ There is growing evidence, however, that diastolic retrograde flow in the descending aorta may be more common than previously thought, especially in patients with atherosclerotic disease. Diastolic retrograde flow in the descending aorta may represent an overlooked mechanism of retrograde embolization in stroke patients. The underlying physiology is related to the increased aortic stiffness due to aortic atherosclerosis. Increased pulse wave velocity and earlier wave reflection at the periphery can then result in marked diastolic retrograde descending aortic flow, even in the absence of aortic valve insufficiency. A number of studies in patients with cryptogenic stroke have shown that a high proportion of these patients demonstrate retrograde embolization of aortic plaque that could reach all aortic branch vessels. ${ }^{33,37,39-42}$

\section{Aortic coarctation}

A number of sequences can and should be used in the preoperative evaluation of aortic coarctation. Morphologic study 
can be obtained with dark blood T1-weighted sequences. Cine imaging with SSFP sequences allow for assessment of morphologic features as well as flow perturbances. ${ }^{43}$ In the setting of aortic coarctation, recent studies suggest that 4D flow imaging can help to reliably calculate collateral flow as well as aortic flow profiles. Three-dimensional flow patterns can be identified that correlate with postrepair complications, including aneurysm and rupture. Four-dimensional flow MRI has provided further insights into the pathophysiology of aortic coarctation as well. ${ }^{44,45}$ Studies have demonstrated altered vessel wall function in coarctation patients, which are largely thought to be secondary to changes in blood flow patterns. These altered flow patterns are thought to result in endothelial dysfunction and arterial remodeling downstream to stenosis. ${ }^{44,45}$ Prior studies have used 4D CE-MRA to assess wall shear stress in the aorta of patients with bicuspid aortic valves as well as those with aortic coarctation. Elevated wall shear stress in the setting of aortic coarctation is thought to predispose these patients to development of ascending aortic aneurysms. ${ }^{46,47}$ Wall shear stresses can be calculated using derivatives of the three-directional blood flow velocities onto a vessel contour. ${ }^{48}$ However, this is computationally intensive and not used on a regular basis.

\section{Mesenteric arteries}

The primary pathologies encountered in imaging of the mesenteric arteries include chronic atherosclerosis, acute embolism, and aneurysm. In the past, conventional angiography was by far the best method for evaluating disease of the mesenteric vasculature. However, MRA is emerging as an effective tool in the radiologist's armamentarium for imaging of the mesenteric vasculature. Non-contrast TOF MRA has been shown to be effective in imaging the proximal portions of the celiac, superior mesenteric, and inferior mesenteric arteries. However, this technique is limited due to long scan times and flow-dependent signal loss from in-plane and turbulent flow. ${ }^{49}$ However, CE-MRA is an excellent tool in evaluation of the mesenteric vasculature. CE-MRA benefits from a high signal-to-noise ratio and short image times. Clinical trials have demonstrated that CE-MRA has sensitivity ranging from $90 \%$ to $100 \%$ when compared with conventional angiography is assessing celiac and superior mesenteric artery disease; however, accuracy is lower for the inferior mesenteric arteries and for the secondary and tertiary branches of the mesenteric vessels..$^{50,51}$

Phase-contrast MRA is also an important tool in evaluation of the splanchnic vasculature. PC-MRA can be used in evaluation of splanchnic perfusion. For example, one study found that patients with chronic mesenteric ischemia have impairment in the normal post-prandial increase in superior mesenteric artery flow when compared with normal controls. ${ }^{52}$ Furthermore, PC-MRA can be used to assess flow perturbances in patients with chronic mesenteric ischemia and can be used to successfully identify those who would benefit from revascularization. ${ }^{53}$

\section{Peripheral arterial disease}

Both CE-MRA and non-contrast MRA are effective tools in the evaluation of peripheral arterial disease. While CTA remains the preferred imaging modality at many centers, CE-MRA is emerging as an effective alternative with many advantages over CTA. CE-MRA has been shown to be equally as accurate in assessment of vascular stenoses within the lower extremity vasculature. ${ }^{54,55}$ One advantage of CE-MRA is that the artifact from bone or calcified plaque is reduced, thus allowing for improved diagnostic accuracy in some instances. The primary technique for peripheral CE-MRA is the bolus chase method. With this technique, contrast is injected and images are obtained in the arterial first-pass phase. Blood pool gadolinium contrast agents with prolonged intravascular stay can be used for first-pass and steady-state MRA. ${ }^{1}$ CE techniques can also be used to obtain time-resolved images. These are valuable in detecting collateral flow pathways, assessing asymmetric flow states and in visualizing arterial to venous shunting. Time-resolved imaging of contrast kinetics (TRICKS) sequences has been shown to provide optimal spatial and temporal resolution. Using TRICKS, multiple images are acquired rapidly to show passage of contrast through the arteries of interest. ${ }^{2}$ Three-dimensional Cartesian CE-MRA with projection-like reconstruction has also been shown to have superior performance when compared with CTA for imaging runoff vessels below the knee (Figure 2). ${ }^{38}$

One disadvantage of CE-MRA is that many patients with peripheral arterial disease suffer from concomitant chronic renal failure. ${ }^{1}$ Thus, exposure to gadolinium-based contrast agents could expose these patients to a risk of nephrogenic systemic fibrosis. Large contrast boluses are required to evaluate the pedal arteries as well. ${ }^{1}$ Thus, non-contrast MRA techniques have emerged as an effective alternative. Non-enhanced, ECG-gated high-resolution QISS MRA is emerging as the preferred non-contrast MRA technique in evaluation of the peripheral vasculature. TOF MRA, PC-MRA, and turbo spin echo MRA have been shown to be inadequate in the assessment of peripheral arterial disease 


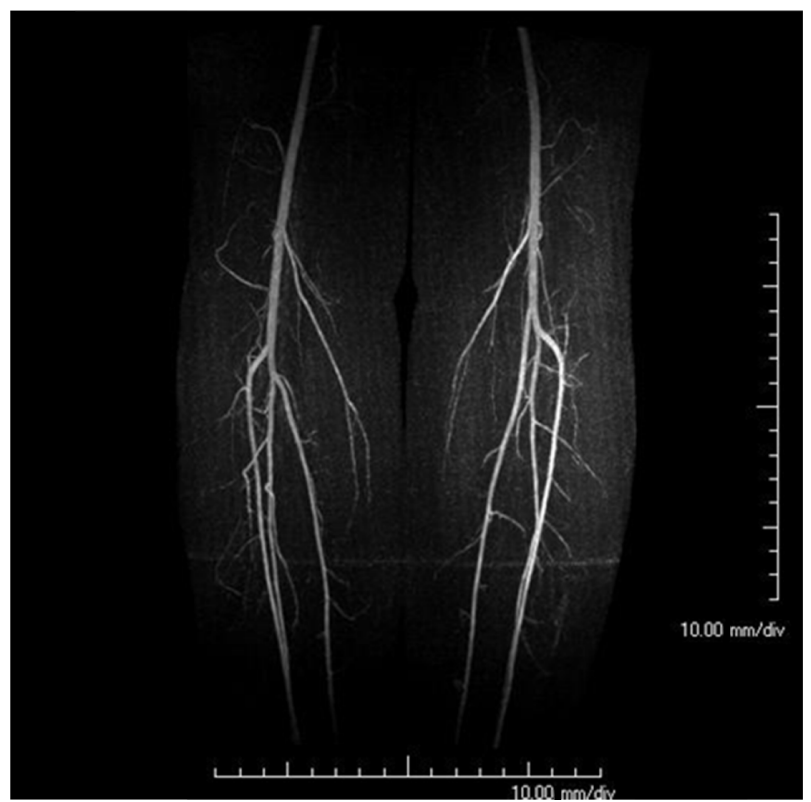

Figure 2 Cartesian acquisition with projection-like reconstruction lower extremity run-off. Time-resolved magnetic resonance angiography of the lower extremities with high spatial and temporal performed using Cartesian acquisition with projectionlike reconstruction.

in many series, while QISS MRA has been shown to have sensitivities and specificities over $90 \%$ when compared with CE-MRA. ${ }^{56}$ High-resolution QISS at high field strengths (3 Tesla) can allow for assessment of very advanced disease. Further, at high field strengths, high-resolution QISS allows for improved contrast between arteries and surrounding soft tissues. In a study comparing CE-MRA with high-resolution QISS, Thierfelder et al found that only 1\% of stenotic segments were able to be assessed on CE-MRA but not high-resolution QISS MRA. ${ }^{56}$ QISS MRA did not suffer from many of the pitfalls of other non-contrast MRA techniques, such as overestimation of degree of stenosis, low image quality, and low diagnostic accuracy for areas of peripheral arterial disease. However, QISS does suffer from poor imaging quality of the distal aorta and the iliac and

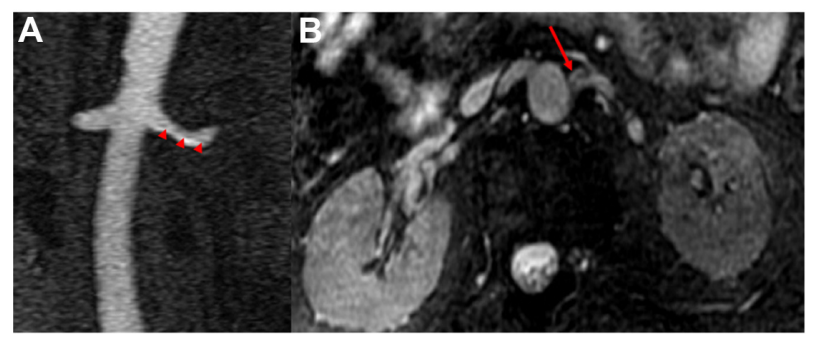

Figure 3 Renal artery dissection.

Notes: (A) Contrast-enhanced magnetic resonance angiography with $1.8 \mathrm{~mm}$ slice thickness demonstrates a subtle dissection flap (red arrowheads). (B) The dissection flap (arrow) is much easier to appreciate on respiratory-navigated vector electrocardiogram-triggered three-dimensional steady-state free precession images. femoral arteries, has long image times, and signal dropout from stents and metal clips. ${ }^{56}$

\section{Renal arteries}

Renal artery stenosis is the most common cause of secondary hypertension, with a prevalence of approximately $5 \%$. This can be secondary to atherosclerotic disease or fibromuscular dysplasia. Early detection and treatment of renal artery stenosis is essential to decrease the risk of loss of renal function and chronic effects of hypertension. Sensitivities and specificities for CE-MRA in the evaluation of renal artery stenosis are upward of $90 \% .{ }^{57}$ However, CE-MRA is limited by bolus profiles and motion. One major limitation of CE-MRA is that many patients with renal artery stenosis also suffer from chronic renal failure, thus making them poor candidates for contrast-enhanced techniques. ${ }^{9}$ Because of this, many non-contrast techniques have emerged. TOF, PC-MRA, and SSFP imaging have been used to evaluate the degree of stenosis with high accuracy. ${ }^{9}$

Three-dimensional TOF techniques have been shown to be fairly accurate in evaluation of renal artery stenosis, with sensitivities of $50 \%-100 \%$ and specificities of $75 \%-100 \%$. Limitations of TOF include long scan times, saturation of in-plane flow, and overestimation of degree of stenosis. This technique is not routinely used in evaluation of renal artery stenosis. PC-MRA images have higher sensitivity than TOF MRA, ranging from $90 \%$ to $100 \%$, with equally high specificity. PC-MRA can be used to determine the hemodynamic significance of a stenosis by evaluation of trans-stenotic pressure drops. Post-stenotic dephasing in PC-MRA has been shown to be associated with the response to revascularization. Further, integrating PC-MRA findings with CE-MRA can result in improved diagnostic accuracy that CE-MRA alone. ${ }^{58}$ Use of SSFP images is the most robust non-contrast imaging technique used for evaluation of renal artery stenosis, with sensitivities and specificities close to $100 \%$ when compared with CE-MRA. ${ }^{59}$ In the setting of renal artery dissection and vasculitides, SSFP images have been shown to be equivalent to CE-MRA (Figure 3). ${ }^{60}$

\section{Conclusion}

A number of MRA techniques are available for evaluation of vascular disease. A number of non-contrast techniques are available that do not expose patients to the potentially toxic effects of gadolinium. Dynamic time-resolved imaging is emerging as an important tool in the evaluation of the hemodynamic consequences of vascular disease. Further research is needed to evaluate some of the newer 4D flow 
MRA techniques and to determine their role in the clinical management of patients. Ultimately, MRA has emerged as an essential tool in improving our understanding of vascular disease.

\section{Disclosure}

The authors report no conflicts of interest in this work.

\section{References}

1. Nielsen YW, Thomsen HS. Contrast-enhanced peripheral MRA: technique and contrast agents. Acta Radiol. 2012;53(7):769-777.

2. Kramer JH, Grist TM. Peripheral MR angiography. Magn Reson Imaging Clin N Am. 2012;20(4):761-776.

3. Zhang H, Maki JH, Prince MR. 3D contrast-enhanced MR angiography J Magn Reson Imaging. 2007;25(1):13-25.

4. Bremerich J, Bilecen D, Reimer P. MR angiography with blood pool contrast agents. Eur Radiol. 2007;17(12):3017-3024

5. Nielsen YJ. Whole-body MR angiography in patients with peripheral arterial disease. Dan Med Bull. 2010;57(12):B4231.

6. Grist TM, Mistretta CA, Strother CM, Turski PA. Time-resolved angiography: past, present, and future. J Magn Reson Imaging. 2012;36(6):1273-1286.

7. Wheaton AJ, Miyazaki M. Non-contrast enhanced MR angiography: physical principles. J Magn Reson Imaging. 2012;36(2):286-304.

8. Miyazaki M, Akahane M. Non-contrast enhanced MR angiography: established techniques. J Magn Reson Imaging. 2012;35(1):1-19.

9. Miyazaki M, Isoda H. Non-contrast-enhanced MR angiography of the abdomen. Eur J Radiol. 2011;80(1):9-23.

10. Uribe S, Hussain T, Valverde I, et al. Congenital heart disease in children: coronary MR angiography during systole and diastole with dual cardiac phase whole-heart imaging. Radiology. 2011;260(1):232-240.

11. Ward EV, Galizia MS, Usman A, Popescu AR, Dunkle E, Edelman RR. Comparison of quiescent inflow single-shot and native space for nonenhanced peripheral MR angiography. J Magn Reson Imaging. 2013;38(6):1531-1538.

12. Clough R, Taylor P. Future imaging techniques in aortic pathologies and clinical implications. J Cardiovasc Surg (Torino). 2013; 54(1 Suppl 1):15-19.

13. Sommer G, Bremerich J, Lund G. Magnetic resonance imaging in valvular heart disease: clinical application and current role for patient management. J Magn Reson Imaging. 2012;35(6):1241-1252.

14. Biglands JD, Radjenovic A, Ridgway JP. Cardiovascular magnetic resonance physics for clinicians: Part II. J Cardiovasc Magn Reson. 2012;14:66.

15. Rengier F, Geisbusch P, Vosshenrich R, et al. State-of-the-art aortic imaging: part I - fundamentals and perspectives of CT and MRI. Vasa. 2013;42(6):395-412.

16. Essig M, Dinkel J, Gutierrez JE. Use of contrast media in neuroimaging. Magn Reson Imaging Clin N Am. 2012;20(4):633-648.

17. Nael K, Moriarty JM, Finn JP. Low dose CE-MRA. Eur J Radiol. 2011;80(1):2-8.

18. Hartung MP, Grist TM, Francois CJ. Magnetic resonance angiography: current status and future directions. J Cardiovasc Magn Reson. 2011; 13:19.

19. Kanal E, Maravilla K, Rowley HA. Gadolinium contrast agents for CNS imaging: current concepts and clinical evidence. AJNR Am J Neuroradiol. May 22, 2014. [Epub ahead of print.]

20. Shokrollahi H. Contrast agents for MRI. Mater Sci Eng C Mater Biol Appl. 2013;33(8):4485-4497.

21. Frydrychowicz A, Lubner MG, Brown JJ, et al. Hepatobiliary MR imaging with gadolinium-based contrast agents. J Magn Reson Imaging 2012;35(3):492-511.

22. Abu-Alfa AK. Nephrogenic systemic fibrosis and gadolinium-based contrast agents. Adv Chronic Kidney Dis. 2011;18(3):188-198.
23. Kanda T, Ishii K, Kawaguchi H, Kitajima K, Takenaka D. High signal intensity in the dentate nucleus and globus pallidus on unenhanced T1-weighted MR images: relationship with increasing cumulative dose of a gadolinium-based contrast material. Radiology. 2014;270(3): 834-841.

24. von Knobelsdorff-Brenkenhoff F, Gruettner H, Trauzeddel RF, Greiser A, Schulz-Menger J. Comparison of native high-resolution 3D and contrast-enhanced MR angiography for assessing the thoracic aorta. Eur Heart J Cardiovasc Imaging. 2014;15(6):651-658.

25. van Bogerijen GH, van Herwaarden JA, Conti M, et al. Importance of dynamic aortic evaluation in planning TEVAR. Ann Cardiothorac Surg. 2014;3(3):300-306.

26. Joseph A, Kowallick JT, Merboldt KD, et al. Real-time flow MRI of the aorta at a resolution of $40 \mathrm{msec}$. J Magn Reson Imaging. 2014;40(1): 206-213.

27. Muzzarelli S, Monney P, O'Brien K, et al. Quantification of aortic flow by phase-contrast magnetic resonance in patients with bicuspid aortic valve. Eur Heart J Cardiovasc Imaging. 2014;15(1):77-84.

28. Garcia J, Markl M, Schnell S, et al. Evaluation of aortic stenosis severity using 4D flow jet shear layer detection for the measurement of valve effective orifice area. Magn Reson Imaging. 2014;32(7):891-898.

29. Hope MD, Hope TA, Crook SE, et al. 4D flow CMR in assessment of valve-related ascending aortic disease. JACC Cardiovasc Imaging. 2011;4(7):781-787.

30. Baliga RR, Nienaber CA, Bossone E, et al. The role of imaging in aortic dissection and related syndromes. JACC Cardiovasc Imaging. 2014;7(4):406-424

31. Sohns JM, Staab W, Menke J, et al. Vascular and extravascular findings on magnetic resonance angiography of the thoracic aorta and the origin of the great vessels. J Magn Reson Imaging. 2014;40(4):988-995.

32. Amano Y, Sekine T, Suzuki Y, Tanaka K, Takagi R, Kumita S. Time-resolved three-dimensional magnetic resonance velocity mapping of chronic thoracic aortic dissection: a preliminary investigation. Magn Reson Med Sci. 2011;10(2):93-99.

33. Francois CJ, Markl M, Schiebler ML, et al. Four-dimensional, flowsensitive magnetic resonance imaging of blood flow patterns in thoracic aortic dissections. J Thorac Cardiovasc Surg. 2013;145(5):1359-1366.

34. Stein E, Mueller GC, Sundaram B. Thoracic aorta (multidetector computed tomography and magnetic resonance evaluation). Radiol Clin North Am. 2014;52(1):195-217.

35. Faber T, Rippy A, Hyslop WB, Hinderliter A, Sen S. Cardiovascular MRI in detection and measurement of aortic atheroma in stroke/TIA patients. J Neurol Disord. 2013;1(4):139.

36. Katsanos AH, Giannopoulos S, Kosmidou M, et al. Complex atheromatous plaques in the descending aorta and the risk of stroke: a systematic review and meta-analysis. Stroke. 2014;45(6):1764-1770.

37. Chhabra L, Niroula R, Phadke J, Spodick DH. Retrograde embolism from the descending thoracic aorta causing stroke: an underappreciated clinical condition. Indian Heart J. 2013;65(3):319-322.

38. Young PM, McGee KP, Bolster B, et al. Magnetic resonance 4D flow reveals unusual hemodynamics associated with aneurysm formation and a possible cause of cryptogenic stroke in a patient with aortic dissection. J Comput Assist Tomogr. 2014;38(2):216-218.

39. Hashimoto J, Ito S. Aortic stiffness determines diastolic blood flow reversal in the descending thoracic aorta: potential implication for retrograde embolic stroke in hypertension. Hypertension. 2013;62(3): $542-549$.

40. Baek JH, Shin DH, Kang CK, Lee YB. Distal subclavian artery occlusion causing multiple cerebral infarcts consequence of retrograde flow of a thrombus? J Cerebrovasc Endovasc Neurosurg. 2013;15(3): 221-224.

41. Harloff A, Simon J, Brendecke S, et al. Complex plaques in the proximal descending aorta: an underestimated embolic source of stroke. Stroke. 2010;41(6):1145-1150.

42. Harloff A, Strecker C, Dudler P, et al. Retrograde embolism from the descending aorta: visualization by multidirectional 3D velocity mapping in cryptogenic stroke. Stroke. 2009;40(4):1505-1508. 
43. Secchi F, Iozzelli A, Papini GD, Aliprandi A, Di Leo G, Sardanelli F. MR imaging of aortic coarctation. Radiol Med. 2009;114(4):524-537.

44. Arzani A, Dyverfeldt P, Ebbers T, Shadden SC. In vivo validation of numerical prediction for turbulence intensity in an aortic coarctation. Ann Biomed Eng. 2012;40(4):860-870.

45. Schubert R. Coarctation of the abdominal aorta: demonstration of a rare anomaly using contrast-enhanced MR-angiography. Vasa. 2009;38(1):66-71.

46. Szopos M, Poussineau N, Maday Y, et al. Computational modeling of blood flow in the aorta-insights into eccentric dilatation of the ascending aorta after surgery for coarctation. $J$ Thorac Cardiovasc Surg. 2014;148(4):1572-1582.

47. Meierhofer C, Schneider EP, Lyko C, et al. Wall shear stress and flow patterns in the ascending aorta in patients with bicuspid aortic valves differ significantly from tricuspid aortic valves: a prospective study. Eur Heart J Cardiovasc Imaging. 2013;14(8):797-804.

48. Stalder AF, Russe MF, Frydrychowicz A, Bock J, Hennig J, Markl M. Quantitative 2D and 3D phase contrast MRI: optimized analysis of blood flow and vessel wall parameters. Magn Reson Med. 2008;60(5):1218-1231.

49. Heiss SG, Li KC. Magnetic resonance angiography of mesenteric arteries. A review. Invest Radiol. 1998;33(9):670-681.

50. Hagspiel KD, Leung DA, Angle JF, et al. MR angiography of the mesenteric vasculature. Radiol Clin North Am. 2002;40(4):867-886.

51. Prince MR, Narasimham DL, Stanley JC, et al. Breath-hold gadolinium-enhanced MR angiography of the abdominal aorta and its major branches. Radiology. 1995;197(3):785-792.

52. Li KC, Hopkins KL, Dalman RL, Song CK. Simultaneous measurement of flow in the superior mesenteric vein and artery with cine phase-contrast MR imaging: value in diagnosis of chronic mesenteric ischemia. Work in progress. Radiology. 1995;194(2):327-330.
53. Wasser MN, Geelkerken RH, Kouwenhoven M, et al. Systolically gated 3D phase contrast MRA of mesenteric arteries in suspected mesenteric ischemia. J Comput Assist Tomogr. 1996;20(2):262-268.

54. Menke J, Larsen J. Meta-analysis: Accuracy of contrast-enhanced magnetic resonance angiography for assessing steno-occlusions in peripheral arterial disease. Ann Intern Med. 2010;153(5):325-334.

55. Chan D, Anderson ME, Dolmatch BL. Imaging evaluation of lower extremity infrainguinal disease: role of the noninvasive vascular laboratory, computed tomography angiography, and magnetic resonance angiography. Tech Vasc Interv Radiol. 2010;13(1):11-22.

56. Thierfelder KM, Meimarakis G, Nikolaou K, et al. Non-contrastenhanced MR angiography at 3 Tesla in patients with advanced peripheral arterial occlusive disease. PLoS One. 2014;9(3):e91078.

57. Vasbinder GB, Nelemans PJ, Kessels AG, Kroon AA, de Leeuw PW, van Engelshoven JM. Diagnostic tests for renal artery stenosis in patients suspected of having renovascular hypertension: a meta-analysis. Ann Intern Med. 2001;135(6):401-411.

58. Auerbach EG, Martin ET. Magnetic resonance imaging of the peripheral vasculature. Am Heart J. 2004;148(5):755-763.

59. Herborn CU, Watkins DM, Runge VM, Gendron JM, Montgomery ML, Naul LG. Renal arteries: comparison of steady-state free precession MR angiography and contrast-enhanced MR angiography. Radiology. 2006;239(1):263-268.

60. Sidhu R, Lockhart ME. Imaging of renovascular disease. Semin Ultrasound CT MR. 2009;30(4):271-288.
Journal of Vascular Diagnostics

\section{Publish your work in this journal}

Journal of Vascular Diagnostics is an international, peer-reviewed journal of diagnostics, focusing on non invasive vascular investigation methods involved in the evaluation of vascular diseases. The journal is committed to the rapid publication in the fields of vascular diseases. Original research, review, case reports, expert opinion and commentaries

\section{Dovepress}

are all considered for publication. The manuscript management system is completely online and includes a very quick and fair peer-review system, which is all easy to use. Visit http://www.dovepress.com/testimonials.php to read real quotes from published authors. 